\title{
Le Régime social britannique à l'heure du Brexit, entre complexité constitutionnelle et incertitude politique
}

The British Welfare Regime in the Context of Brexit, amidst Constitutional

Complexity and Political Uncertainty

\section{Clémence Fourton}

\section{(2) OpenEdition}

Journals

Édition électronique

URL : https://journals.openedition.org/rfcb/7808

DOI : $10.4000 /$ rfcb.7808

ISSN : 2429-4373

Éditeur

CRECIB - Centre de recherche et d'études en civilisation britannique

Référence électronique

Clémence Fourton, « Le Régime social britannique à l'heure du Brexit, entre complexité constitutionnelle et incertitude politique », Revue Française de Civilisation Britannique [En ligne], XXVI-2 I 2021, mis en ligne le 05 janvier 2021, consulté le 04 janvier 2022. URL : http:// journals.openedition.org/rfcb/7808; DOI : https://doi.org/10.4000/rfcb.7808

Ce document a été généré automatiquement le 4 janvier 2022.

\section{c) $(1) \odot$}

Revue française de civilisation britannique est mis à disposition selon les termes de la licence Creative Commons Attribution - Pas d'Utilisation Commerciale - Pas de Modification 4.0 International. 


\title{
Le Régime social britannique à l'heure du Brexit, entre complexité constitutionnelle et incertitude politique
}

\author{
The British Welfare Regime in the Context of Brexit, amidst Constitutional \\ Complexity and Political Uncertainty
}

Clémence Fourton

\section{Introduction}

1 En 1990, Gøsta Esping-Andersen change durablement l'analyse de l'État social en lui substituant la notion de régime social, dans son ouvrage The Three Worlds of Welfare Capitalism ${ }^{1}$. Cette proposition épistémologique allonge considérablement la liste des objets d'étude pour quiconque s'intéresse aux politiques sociales d'un pays : au-delà des institutions sociales à proprement parler, comme le National Health Service, le régime social inclut aussi les normes qui les sous-tendent, les discours qui s'y rattachent, les effets qu'elles produisent. En somme, le régime social inclut tout ce qui détermine, et qui est déterminé par l'État social au sens strict. Prendre au sérieux cette épistémologie implique alors aussi, en retour, de considérer ce qui contribue à façonner le welfare state sans pour autant relever du social proprement dit. C'est à ce titre que cet article se penche sur la matrice constitutionnelle de l'État social britannique, à l'heure où celle-ci est transformée par la sortie du Royaume-Uni de l'Union européenne (UE).

Il va sans dire que les développements qui font l'objet de cette étude sont, précisément, des développements, et qu'à ce titre ils sont récents, voire en cours, et, en tout cas, volatiles. Nous faisons l'hypothèse que cette volatilité n'empêche pas l'analyse, pourvu que l'on ne lui demande pas plus que ce qu'elle peut fournir. Les analyses qui suivent n'ont pas de valeur prédictive ; il s'agit, à la lumière des dynamiques institutionnelles, 
juridiques et politiques qui marquent le Royaume-Uni dans le contexte du Brexit, mais aussi avant celui-ci, de dégager des points saillants et des tendances quant à l'articulation entre changements constitutionnels et changements sociaux. En cela, ce texte est, plus qu'un diagnostic du régime social à l'heure du Brexit, une invitation à observer le Brexit par le prisme du régime social.

3 Au cœur de l'articulation entre arrangements constitutionnels et dispositions sociales se trouve la question de la citoyenneté telle que conceptualisée par Thomas Marshall dès $1950^{2}$ - une conception tridimensionnelle de la citoyenneté, non seulement comme civile et politique, mais aussi sociale. Que change donc le Brexit pour la citoyenne de Marshall, c'est-à-dire pour une Britannique qui n'est plus européenne, et qui est usagère d'un système de santé, d'un système de protection sociale, d'un système éducatif auxquels les normes européennes ne s'appliquent plus? En d'autres termes, considérer l'articulation entre Brexit et welfare state amène à se pencher sur les effets $\mathrm{du}$ premier en termes de définition et d'accès au second; en cela, la question du contenu social de la démocratie est au cœur de notre réflexion, qui procède en trois temps. Dans une première partie, on se concentre sur les effets de la sortie du Royaume-Uni de l'UE sur les institutions sociales du pays. Ensuite, on se tourne vers les conséquences juridiques du Brexit, en termes de droits sociaux pour les Britanniques. Enfin, la troisième partie propose d'analyser comment les changements constitutionnels en cours s'articulent avec les orientations des gouvernements britanniques en matière de politique sociale.

\section{Brexit et institutions sociales}

4 Le bouleversement constitutionnel que constitue la sortie du Royaume-Uni de l'UE ne peut être examiné, en matière d'institutions sociales, indépendamment d'un autre changement qui marque le paysage constitutionnel britannique depuis la fin des années 1990 : la dévolution, autrement dit la décentralisation du pouvoir législatif et exécutif depuis le parlement et le gouvernement britanniques vers d'autres niveaux de pouvoir. Vers les nations écossaises et galloises et la province d'Irlande du Nord à partir de $1998^{3}$, puis vers certaines métropoles ${ }^{4}$. Au niveau des nations, sur lequel on se concentre ici, en dépit de différences quant au processus d'identification des domaines dévolus et réservés dans les trois territoires, les domaines in fine dévolus sont d'une remarquable homogénéité entre les trois gouvernements: les compétences dévolues concernent principalement les politiques sociales, si bien que la dévolution a pu être analysée comme la création de trois parlements dédiés à la politique sociale (social policy parliaments ${ }^{5}$ ). Ainsi, les domaines qui relèvent de l'État social figurent presque tous au rang des compétences dévolues : santé, éducation, logement, sport et culture, transports... Avec une exception notable, qui est celle de la législation portant sur le travail (sauf pour l'Irlande du Nord) et, dans une certaine mesure, la protection sociale, c'est-à-dire les aides financières de l'État aux individus (retraites, chômage, allocations familiales...). La dévolution a donc créé des possibilités de différenciation en matière d'institutions sociales, qui sont à comprendre à la fois à l'échelle de l'Écosse, du pays de Galles et de l'Irlande du Nord, et dans leurs rapports avec les politiques britanniques ${ }^{6}$.

5 La dévolution revêt alors une importance toute particulière dans le contexte du Brexit, car l'UE était l'un des facteurs de convergence entre les institutions sociales des différents territoires du Royaume-Uni : Derek Birrell estime ainsi que les trois-quarts 
des prérogatives du gouvernement écossais étaient, avant la sortie britannique de l'UE, influencées par des décisions prises à Bruxelles ${ }^{7}$. En dépit de ce facteur de convergence, deux éléments de différenciation en matière d'institutions sociales sont à noter à l'échelle des nations : la mise en avant, au pays de Galles et en Écosse, du principe d'universalité dans les politiques sociales ${ }^{8}$ et dans une moindre mesure dans l'éducation', ainsi que la part du secteur privé dans le fonctionnement des services d'intérêt collectif.

6 Par exemple, concernant les aides financières aux individus, le parlement écossais a adopté, dans la foulée du Welfare Reform Act britannique de 2012 une loi ${ }^{10}$ permettant une compensation, au niveau de l'Écosse, de la baisse de certaines aides sociales décidée au niveau britannique. De même, la dynamique de marchandisation du système de santé, tandis qu'elle va crescendo en Angleterre, tend au contraire à régresser dans les autres territoires britanniques depuis l'introduction de la dévolution. Ainsi, si en Irlande du Nord le principe de mise en concurrence de différentes composantes du NHS (ce qu'on appelle le "marché interne ») est également en vigueur, mais soumis à une plus forte régulation ${ }^{11}$, dans les deux autres nations, celui-ci a été tout simplement aboli - en Écosse en 2004 puis au pays de Galles en 2009. Outre le fait que, une fois hors de l'UE, les différentes nations britanniques ne seront plus soumises au même cadre législatif communautaire et que donc, ces différenciations sont susceptibles de s'accentuer, deux autres questions se posent : une question économique et une question de gouvernance.

7 Premièrement, il y a la question économique des fonds européens jusque là alloués au Royaume-Uni, dont le Brexit signe l'interruption. Parmi eux figurent les Fonds structurels et d'investissement européens et le Fonds européen agricole de garantie, qui sont administrés directement par les nations britanniques. Le Fonds européen agricole de garantie constitue le premier pilier de la Politique Agricole Commune (PAC) et a représenté 22,5 milliards d'euros sur la période 2014-2020, versés directement aux agriculteurs britanniques ${ }^{12}$. Les Fonds structurels et d'investissement européens, quant à eux, comptent notamment le Fonds européen de développement régional, le Fonds social européen, et le Fonds européen agricole pour le développement rural (connu comme le second pilier de la PAC) et ont représenté 17,5 milliards d'euros sur la période 2014-2020. Or, ces Fonds structurels et d'investissement européens sont attribués en fonction du développement économique régional: si l'on ramène les totaux à la population, sur la même période, le pays de Galles est de loin le plus subventionné, à hauteur de $140 €$ par habitant(e), devant l'Irlande du Nord (57€), l'Écosse (47€) et l'Angleterre $(27 €)^{13}$. Dans ces conditions, le gouvernement britannique sera-t-il en mesure de suppléer aux budgets reçus par les nations via ces fonds, et si oui, selon quelles modalités?

8 La question du remplacement de ce manque à gagner, à l'issue de la période de transition, n'est pas tranchée. Si la formule de Barnett, habituellement employée par le gouvernement britannique pour établir les enveloppes budgétaires dévolues aux nations, était utilisée dans la perspective d'une compensation britannique des fonds européens, elle substituerait aux politiques européennes, basées sur les inégalités régionales et donc les besoins spécifiques des nations et régions, une logique basée sur le nombre d'habitant(e)s. Alan Greer souligne donc que le Brexit agit là comme le révélateur des limites des arrangements constitutionnels en vigueur, et que la formule de Barnett doit être, sinon abandonnée, du moins réévaluée ${ }^{14}$. De fait, le Brexit, s'il n'est 
pas voué à accentuer les inégalités socio-spatiales britanniques, vient rappeler leur existence, et l'absence de prise en compte de celles-ci dans les mécanismes budgétaires liés à la dévolution.

Deuxièmement, en matière de gouvernance, se pose la question du rapatriement des compétences jusque là gérées au niveau des institutions européennes et qui sont ellesmêmes, à l'échelle du Royaume-Uni, dévolues aux nations. En matière d'agriculture, de pêche, de commerce et d'environnement, les nations vont donc être en mesure de mettre en œuvre des politiques susceptibles de s'écarter de l'orientation non seulement communautaire, mais aussi britannique. Dans cette perspective, le gouvernement britannique a évoqué la nécessité d'identifier des "cadres communs" (common frameworks), à l'échelle du Royaume-Uni, pour limiter les différenciations territoriales. Des orientations générales ont été posées: il s'agit notamment d'assurer le fonctionnement du marché britannique, de respecter les engagements internationaux du pays, et d'optimiser la gestion des ressources ${ }^{15}$. Là encore, la question des inégalités régionales se pose. D'une part, une augmentation des prérogatives des nations qui ne serait pas corrélée à une marge de manœuvre budgétaire accrue les laisserait finalement incapables de mener des politiques à la hauteur de leurs besoins spécifiques. D'autre part, la définition des cadres communs fait, à l'échelle britannique, l'objet de négociations dont l'issue dépendra du rapport de force à l'œuvre entre les gouvernements nationaux et le gouvernement britannique. Or, ce dernier est par définition en position de force, puisque l'existence-même de la dévolution peut être remise en cause par la législation britannique - et si cette proposition peut sembler irréaliste, elle ne l'est sans doute pas plus que ne l'était la perspective d'une sortie de l'Union européenne il y a quelques années.

10 Ainsi, la sortie de l'UE vient se combiner à des arrangements constitutionnels préexistants, au premier rang desquels la dévolution, qui a été mise en œuvre dans un contexte où la participation à l'UE était pensée comme pérenne. Dans ces conditions, le Brexit vient renforcer la dynamique initiée par la dévolution en matière d'institutions sociales. D'une part, la dévolution est une force centrifuge, puisqu'une partie du pouvoir exécutif et législatif est transférée au niveau des nations qui composent le Royaume-Uni. D'autre part, l'UE peut être vue comme une force centripète, puisqu'elle a un effet d'homogénéisation légale et politique sur ses territoires. Cette homogénéisation passe également par une prise en compte des disparités économiques et sociales entre les nations, puisque l'UE alloue des fonds de développement aux régions les plus en difficulté. Certes, les contributions versées par le Royaume-Uni à l'UE étaient plus importantes que les subventions qu'il en recevait - 9,2 milliards d'euros de contributions par an en moyenne entre 2010 et 2017, contre 6,8 milliards d'euros de subventions ${ }^{16}$. Mais l'UE jouait ici un rôle redistributif, en favorisant les anciennes régions industrielles galloises et la Cornouailles en premier lieu, par rapport à d'autres régions plus dynamiques. Quitter l'UE, c'est donc aussi, pour le Royaume-Uni, renoncer à cette dimension non seulement unificatrice mais aussi redistributive. Le Brexit est donc susceptible de produire une différenciation accrue des institutions sociales à l'échelle des territoires britanniques, ce qui en soi ne présage en rien de la forme de cette différenciation. En revanche, il est certain que cette différenciation prendra la forme d'une aggravation des inégalités territoriales si la prise en compte de celles-ci n'est pas intégrée au fonctionnement et au financement des institutions sociales du pays. 


\section{Brexit et droits sociaux}

11 du 23 juin 2016, date du référendum, au 31 janvier 2020, date de sortie de l'UE, deux lois revêtent une importance particulière quant à la question de l'impact de la sortie de l'UE sur les droits sociaux des Britanniques ${ }^{17}$. La première de ces deux lois est appelée European Union (Withdrawal) Act 2018, ou Loi sur la sortie de 2018, et la seconde est la loi adoptée le 23 janvier 2020, baptisée European Union (Withdrawal Agreement) Act, ou Loi sur l'accord de sortie de 2020. La seconde de ces lois dérive directement de la première, qui impose au gouvernement britannique d'obtenir l'aval du parlement pour la ratification de l'accord de sortie, et vient la compléter et remplacer certaines de ses dispositions ${ }^{18}$. période de transition (entre le 31 janvier 2020 et le 31 décembre 2020), et des dispositions de long terme, notamment quant à la mise en œuvre de l'Accord de sortie qui doit être signé par le Royaume-Uni et les différents pays de l'UE avant la fin de la période de transition : celui-ci sera contraignant pour la législation britannique future. La Loi sur la sortie de 2018, prévoit, quant à elle, d'une part l'abrogation de la législation en vertu de laquelle le Royaume-Uni était membre de l'UE, et statue d'autre part sur le devenir du droit européen affectant le Royaume-Uni. Une partie des normes européennes en vigueur au Royaume-Uni sont ainsi incorporées à la législation britannique; elles sont "conservées» (retained) pour assurer une continuité législative ${ }^{19}$.

13 L'incorporation du droit européen comporte plusieurs exceptions : d'abord, le principe de supériorité du droit européen sur le droit britannique, s'il est conservé pour les textes datant d'avant le 31 décembre 2020, ne s'appliquera plus après cette date autrement, la loi britannique serait restée « subordonnée » au droit communautaire, et le Royaume-Uni n'aurait pas retrouvé son autonomie législative. Ensuite, et c'est un élément majeur pour notre étude, la Charte des droits fondamentaux de l'Union européenne, signée en 2000, intégrée au Traité de Lisbonne de 2007, et à l'application de laquelle veille la Cour de justice de l'Union européenne, n'est pas transférée en tant que telle dans le droit britannique. En revanche, le Royaume-Uni est un des membres fondateurs du Conseil de l'Europe, créé en 1949 et a été le premier pays à ratifier, en 1950, la Convention européenne des droits de l'Homme, dont la Cour européenne des droits de l'Homme veille au respect du contenu. En 1998 a été adoptée au Royaume-Uni la loi Human Rights Act, qui incorpore en droit national la Convention. Cette transposition en droit britannique, si elle ne s'est pas faite sans controverses, a intégré le régime des droits de l'homme au droit national, et a provoqué un développement du langage des droits de l'homme dans le discours politique britannique ${ }^{20}$.

14 Pourtant, le fait que le Royaume-Uni ne remette pas en cause - pour le moment - sa ratification de la Convention ne constitue pas une garantie des droits des citoyen(ne)s britanniques à hauteur de celle apportée par la Charte, et ce pour deux raisons. La première est que la Convention ne porte pas sur les mêmes droits que la Charte : il y figure moins de domaines, et pour les domaines qui sont représentés dans les deux textes, les droits de la Convention sont moins détaillés que ceux contenus dans la Charte. Par exemple, l'Article 31 de la Charte précise que «Tout travailleur a droit à des 
conditions de travail qui respectent sa santé, sa sécurité et sa dignité » tandis qu'il n'y a rien à ce sujet dans la Convention. Les deux textes, en revanche, comportent des éléments sur la liberté de réunion et d'association. La Convention indique que toute personne a droit à la liberté de réunion pacifique et à la liberté d'association ; la Charte étend les droits à la liberté de réunion pacifique et à la liberté d'association à tous les niveaux, et précise que sont notamment concernés le domaine politique et syndical.

La seconde faiblesse de la Convention par rapport à la Charte est que les procédures légales utilisables par les citoyen(ne)s pour faire appliquer les droits présents dans la Convention sont moins robustes que celles liées à la Charte ${ }^{21}$. Les citoyen(ne)s britanniques ne pourront plus s'en remettre à la Cour de justice de l'Union européenne au sujet des droits sociaux présents dans la Charte et qui seraient bafoués. En somme, la Charte jouait, pour les citoyen(ne)es britanniques, l'un des rôles d'une constitution formalisée, au sens où elle constituait un document unique, opposable, dans lequel figuraient les droits qui leur incombaient. Elle représentait notamment une garantie pour les citoyen(ne)s située en-dehors du parlement britannique, et donc des changements de majorité politique. En l'absence d'une constitution rédigée, et selon la doctrine de la souveraineté parlementaire, tout parlement britannique peut en effet modifier les droits des citoyen(ne)es au moyen d'une simple loi.

Pour la citoyenne britannique, la sortie de l'UE équivaut donc à une perte de garanties juridiques en matière de droits sociaux, d'autant plus qu'elle perd le bénéfice de la jurisprudence de la Cour de justice de l'Union européenne sur ceux-ci. Si le fonctionnement des tribunaux britanniques n'empêche en théorie pas les juges de continuer à se servir de la Charte des droits fondamentaux de l'Union européenne pour rendre leurs jugements, rien ne les oblige à le faire. Il faut ajouter à cela un enjeu de taille : en Irlande du Nord, les Accords de Belfast garantissent une égalité des droits entre les citoyen(ne)es britanniques d'Irlande du Nord et les citoyen(ne)s irlandais(e)s de la République d'Irlande. Or, l'Irlande est toujours membre de l'UE, et la Charte s'y applique. Dans ces conditions, soit les Irlandais(e)s bénéficient d'une protection juridique dont les Nord-irlandais(e)s sont privé(e)s à l'issue du Brexit, soit les Nordirlandais(e)s bénéficient d'une protection juridique dont les habitant(e)s de GrandeBretagne sont privé(e)s. Dans les deux cas, il y a une rupture d'égalité susceptible d'avoir des conséquences politiques majeures ${ }^{22}$.

\section{Brexit et politiques sociales}

17 À partir du $1^{\mathrm{er}}$ janvier 2021, le parlement britannique sera en mesure d'adopter des lois qui pourront éloigner progressivement le droit britannique des directives communautaires, dans les limites fixées par l'Accord de sortie qui aura été ratifié par les vingt-sept et le Royaume-Uni ${ }^{23}$. Dans ce contexte, le discours programmatique du gouvernement nouvellement élu de Boris Johnson, le 19 décembre 2019, revêt une importance particulière. Comme tous les Discours de la Reine (Queen's Speech), il identifie les domaines des politiques publiques qui constitueront les priorités du gouvernement au cours de son mandat. Mais dans ce cas précis, il indique aussi quels sont les domaines du droit européen incorporé au droit britannique qui seront prioritairement examinés, et, le cas échéant, modifiés. En somme, il identifie les domaines sur lesquels le gouvernement a l'intention d'exercer dès que possible, sur le plan politique, le « contrôle repris » à l'UE. 

les mois suivants la sortie de l'UE sont nombreux. Figurent parmi les priorités du gouvernement Johnson le soin à la personne, les aides sociales et en particulier les retraites, l'éducation, le handicap, le logement, la justice, la famille, la recherche, les transports et les infrastructures ${ }^{24}$. Les projets sur la santé sont particulièrement développés dans le Discours, de même que le droit du travail. Le fait que le gouvernement ait l'intention de réformer le droit du travail européen incorporé à la législation britannique - qui comprend des dispositions quant à la réglementation du temps de travail, aux congés payés, aux droits liés à la parentalité, et la protection contre la discrimination - n'est pas, en soi, significatif quant au contenu futur de ces modifications : le Royaume-Uni pourrait même choisir d'étendre ces droits. On pourrait ainsi imaginer qu'une fois affranchi du cadre réglementaire de l'UE sur le marché commun, le Royaume-Uni choisisse d'augmenter les régulations portant sur les conditions de travail, et les cotisations sociales. Mais ces développements sont très improbables à l'aune de la coloration résolument néolibérale des politiques de l'emploi adoptées ces dernières années ${ }^{25}$.

19 Là encore, la combinaison du Brexit à la dévolution est susceptible, selon l'échelle de gouvernance qui se verra attribuer la gestion des compétences rapatriées de l'UE de renforcer, ou à l'inverse de tempérer les orientations politiques britanniques. À ce titre, il est à noter que l'adoption de la Loi sur la sortie de 2018 et de la Loi sur l'accord de sortie de 2020 s'est faite, au parlement britannique, sans tenir compte de l'absence de consentement législatif de la part des nations - de l'Écosse dans le premier cas, et de tous les territoires dans le second. Cette situation a donc de facto consisté en un nonrespect de la Convention Sewel, en vertu de laquelle les lois britanniques concernant des domaines dévolus aux nations ne sont pas adoptées sans leur consentement législatif, et laisse à penser que Londres est susceptible d'essayer d'infléchir le rapatriement des compétences européennes dans le sens d'une re-concentration du pouvoir. L'un des facteurs explicatifs à cela est le jeu des équilibres politiques entre les différentes composantes du Royaume-Uni au cours de la dernière décennie.

En effet, depuis 2010, les gouvernements écossais (mené par les nationalistes du Scottish National Party depuis 2007) et gallois (mené par les travaillistes depuis les premières heures de la dévolution) ont constitué une force d'opposition aux gouvernements britanniques successifs, dominés par les conservateurs. La politique sociale s'est alors imposée comme un champ permettant aux nations de se distinguer des politiques sociales menées depuis Londres, et de modérer leur dimension néolibérale, donnant naissance à une forme de nationalisme social (welfare nationalism ${ }^{26}$ ) à l'échelle écossaise, et dans une moindre mesure à l'échelle galloise.

21 Outre les dynamiques politiques des deux dernières décennies, les débats qui ont accompagné l'adoption de la Loi sur l'accord de sortie de 2020 donnent également des indications quant à l'orientation politique que le gouvernement Johnson entend donner au surcroît de marge de manœuvre en matière sociale que lui confère le Brexit. Ainsi, concernant la réforme du droit du travail à venir, baptisée Employment Bill, tout porte à croire qu'elle n'ira pas dans le sens d'une protection accrue des travailleurs et travailleuses britanniques. En effet, la Loi sur l'accord de sortie adoptée le 23 janvier 2020 diffère en un certain nombre de points du projet de loi sur le même sujet, présenté au parlement en octobre 201927. L'une de ces divergences est significative en termes de protection des droits des travailleurs et travailleuses : la loi du 23 janvier 2020 n'inclut 
pas la clause 34 et le planning 4 , à savoir une clause de non-régression en matière de droit du travail. Cela signifie qu'une fois hors de l'UE, la législation britannique n'aura non seulement plus à respecter les standards minimums imposés par l'UE sauf si cela figure dans l'Accord de sortie, mais que ses évolutions n'iront pas non plus nécessairement dans le sens d'une progression des droits des travailleurs et travailleuses.

Dans ces conditions, l'Accord de sortie qui sera signé avec l'UE et qui fixera un cadre pour les réformes futures de l'acquis communautaire au Royaume-Uni pourrait alors constituer un garde-fou pour le droit du travail britannique. La protection des travailleurs et travailleuses est en outre un enjeu crucial des négociations menées entre le Royaume-Uni et l'UE, et cela pour des raisons pragmatiques. En effet, la régulation du marché du travail se doit, dans l'optique du maintien de relations commerciales étroites entre les deux parties, d'être homogène. C'est en tout cas un engagement posé par les deux parties dans la déclaration politique fixant le cadre des relations futures entre l'UE et le Royaume-Uni, prélude aux négociations en vue de trouver un accord de sortie définitif. Toutefois, une note gouvernementale à ce sujet, révélée par le Financial Times $^{28}$ indique clairement que le gouvernement britannique veut minimiser les contraintes liées à l'alignement réglementaire (level playing field ${ }^{29}$ ). Ces signaux indiquent donc une ambition politique de déréglementation accrue du monde du travail, qui suscitent des réactions hostiles. Du côté des acteurs européens, par ailleurs perçus par une partie de la classe politique française comme laxistes en matière de réglementation sociale, on se dit inquiet de ce que le Royaume-Uni, en déviant des standards européens, ne bénéficie d'un avantage compétitif ${ }^{30}$. Du côté des syndicats britanniques, réunis au sein du Trade Union Congress, on a dès 2016 publié un rapport alarmiste sur les effets du Brexit sur le droit du travail ${ }^{31}$. Il apparaît donc que les modifications majeures du fonctionnement de l'État qu'induit le Brexit, dans un contexte de politiques néolibérales d'effritement du welfare state, produit une incertitude quant à la préservation des droits sociaux des Britanniques. Cette incertitude n'est pas seulement celle des observateurs des développements en cours, elle s'applique aussi à la population britannique, pour qui le processus de sortie de l'UE provoque nécessairement un manque de lisibilité quant à la définition à moyen et long terme du régime social qui est le leur.

\section{Conclusion}

Au terme de cette étude des effets de la sortie de l'UE sur le régime social britannique, deux conclusions s'imposent. Premièrement, avec le Brexit, des droits sociaux sont susceptibles d'être affaiblis par des réformes postérieures à l'incorporation en droit britannique du droit européen, du fait de l'absence de garde-fous constitutionnels : une fois hors de la juridiction de la Charte des droits fondamentaux de l'Union européenne, aucune loi fondamentale ne garantit les droits eux-mêmes, ou les changements susceptibles de les affecter, et la Cour de justice de l'Union européenne ne sera plus un recours possible pour les Britanniques. En l'absence de constitution, c'est donc sur les décisions judiciaires nationales et sur les forces politiques britanniques que repose la garantie des droits sociaux. Ces droits vont donc être, dans les prochaines années, davantage soumis aux alternances gouvernementales, et aux logiques d'opposition qui 
se jouent entre le pouvoir britannique central et les gouvernements gallois, écossais et nord-irlandais.

Deuxièmement, le fait que la dévolution ait commencé après l'entrée du Royaume-Uni dans l'UE en 1973 implique que les institutions dévolues n'ont, jusqu'ici, jamais fonctionné en dehors du cadre communautaire, qui fournissait une législation supranationale, commune au Royaume-Uni et à ses différentes composantes. Dans ces conditions, deux dynamiques s'enclenchent: d'une part, la possibilité d'une différenciation accrue des modèles sociaux à l'intérieur du pays, et, d'autre part, la possibilité d'une re-concentration du pouvoir à Londres à l'occasion du rapatriement de compétences européennes. Ces deux dynamiques peuvent se cumuler, puisque, face à ce qui peut être perçu comme un abus du pouvoir central, les gouvernements d'Écosse, du pays de Galles et d'Irlande du Nord sont susceptibles d'utiliser, en réponse, au maximum leurs possibilités de contre-pouvoir. Les électorats entrent aussi en jeu, puisque les tensions constitutionnelles autour de la dévolution peuvent se traduire par des victoires politiques nationalistes. Derrière ces tensions, c'est aussi l'égalité de traitement entre tou(te)s les citoyen(ne)s britanniques qui est en jeu, et la prise en compte des inégalités socio-spatiales. Il y a donc, dans la superposition de la dévolution et de la sortie de l'UE, une forme de paradoxe. Paradoxe car la présence du RoyaumeUni dans l'UE, si elle était source de divisions politiques importantes, notamment entre les nations, était aussi une source d'unité, dans les politiques mises en œuvre, et de compensation des inégalités territoriales. À l'inverse, la sortie de l'UE va très certainement accentuer encore les différences nationales au sein du Royaume-Uni, alors même que le renforcement d'une entité politique britannique était l'un des arguments avancés en faveur du Brexit.

Aux effets strictement législatifs et constitutionnels du Brexit susceptibles de modifier le régime social britannique, il faut ajouter les effets macro-économiques de l'ensemble de la séquence ${ }^{32}$ : les projections économiques, si elles varient dans les chiffres, pointent toutes un ralentissement de l'économie britannique lié au Brexit. Or, une baisse de la croissance entrainera aussi une baisse des revenus de l'impôt, et donc des fonds publics susceptibles d'être alloués aux services publics. De plus, les restrictions migratoires que les Britanniques mettent en place posent une sérieuse question de main d'œuvre, notamment dans le secteur de la santé, du soin à la personne, et dans l'enseignement supérieur et la recherche.

Finalement, la sortie du Royaume-Uni de l'UE agit donc comme un révélateur à bien des niveaux: économique - puisque l'intégration des économies continentales et britanniques est désormais indubitable; politique - puisque les lignes de fracture internes au pays sont exacerbées ; social - puisque le vote Leave en 2016 est directement corrélé à de faibles revenus et un faible niveau d'éducation; territorial - puisque la disparition des fonds européens de soutien aux régions les plus défavorisées oblige Londres à trancher quant à leur remplacement; et constitutionnels - puisque les institutions britanniques, entre dévolution et intégration européenne, fonctionnaient jusqu'en 2020 selon des équilibres d'autant plus fragiles qu'ils ne sont pas codifiés dans un texte constitutionnel fondamental. Cela vaut tant pour les rapports entre les différents niveaux de l'État que pour les rapports des citoyen(ne)s à ceux-ci. C'est donc vers une politisation accrue des questions sociales que l'on se dirige dans les prochaines années; des rapports de force politiques dépendra le visage du régime social britannique hors de l'Union européenne. 


\section{BIBLIOGRAPHIE}

BIRRELL Derek, The Impact of Devolution on Social Policy, Bristol, Policy Press, 2009.

BRIEN Philip, UK funding from the $E U$, Briefing Paper $n^{\circ} 7847$, House of Commons Library, 28 novembre 2018.

Cousson Anne, Droits de l'homme au Royaume-Uni entre 1998 et 2010 : entre politique nationale et droit international, Thèse de doctorat, Université Sorbonne Paris Cité, 2016.

COWIE Graeme, The status of "retained EU law », Briefing Paper n08375, House of Commons Library, 30 juillet 2019.

COWIE Graaeme, DE MARS, Sylvia, KELLY, Richard et TORRANCE, David, Constitutional implications of the Withdrawal Agreement legislation, Briefing Paper n08805, House of Commons Library, 20 février 2020.

DE MARS Sylvia, MURRAY Colin, o'DONOGHUE Aoife et WARWICK Ben, Continuing EU citizenship " rights, opportunity and benefits » in Northern Ireland after Brexit, Belfast, Northern Ireland Human Rights Commission, mars 2020.

DUCOURTIEUX Cécile et PETITJEAN Sophie, « Négociations du Brexit : Londres prêt à tout face à l'UE », Le Monde, 26 février 2020, https://www.lemonde.fr/international/article/2020/02/26/ negociations-du-brexit-londres-pret-a-tout-face-a-l-ue_6030900_3210.html, consulté le 9 avril 2020.

ESPING-ANDERSEN Gøsta, The Three Worlds of Welfare Capitalism, Princeton, Princeton University Press, 1990.

FERGUSON Daniel, « Removal of workers' rights in the new EU (Withdrawal Agreement) Bill », House of Commons Library, 20 décembre 2019, https://commonslibrary.parliament.uk/brexit/ legislation/workers-rights-and-the-new-eu-withdrawal-agreement-bill/, consulté le 9 avril 2020. FORD Michael, Workers' rights from Europe : the impact of Brexit, Report for the Trade Union Congress, 10 mars 2016, https://www.tuc.org.uk/research-analysis/reports/workers-rightseurope-impact-brexit, consulté le 11 avril 2020.

GREER Alan, « Brexit and Devolution », Political Quaterly, n89/1, 2018, p. 134-138.

JEFFREY Charlie, LODGE Guy, et SCHMUECKER Katie, « The devolution paradox », in LODGE Guy et SCHMUECKER Katie (dir.), Devolution in Practice 2010, London, Institute for Public Policy Research, 2010, p. 9-31.

JOZEPA Ilze, FELLA Stefano, BRowning Steve, SEELY Antony, FERGUSON Daniel, SMITG Louise et PRIESTLEY Sara, The UK-EU future relationship negociations : Level playing field, Briefing Paper $n^{\circ} \mathrm{CPB} 8852$, House of Commons Library, 17 mars 2020.

LOCK Tobias, « Human Rights Law in the UK after Brexit », Public Law, novembre 2017, p. 117-134. MARSHALl Thomas H., Citizenship and Social Class, Cambridge, Cambridge University Press, 1964 (1950).

PICKARD Jim et BRUNSDEN Jim, "Fears rise over post-Brexit workers' rights and regulations », The Financial Times, 25 octobre 2019, https://www.ft.com/content/5eb0944e-f67c-11e9-9ef3eca8fc8f2d65, consulté le 9 avril 2020. 
THE QUEEN'S SPEECH, Prime Minister's Office, London, 19 décembre 2019.

SARGEANT Jess et KLEMPERER David, « Devolution : common frameworks and Brexit », Institute for Governement, 16 mars 2020, https://www.instituteforgovernment.org.uk/explainers/devolutioncommon-frameworks-brexit, consulté le 13 avril 2020.

STEWART Kitty, COOPER Kerris et SHUTES Isabel, What does Brexit mean for social policy in the UK?, Social Policies and Distributional outcomes in a Changing Britain, Research Paper $n^{\circ} 3$, février 2019.

TAYLOR-GOOBY Peter, « Re-doubling the crises of the welfare state : the impact of Brexit on UK welfare politics ", Journal of Social Policy, n46/4, 2017, p. 815-835.

THIMONT JACK Maddy, « Withdrawal Agreement Act », Institute for Governement, 4 février 2020, https://www.instituteforgovernment.org.uk/explainers/withdrawal-agreement-act, consulté le 9 avril 2020.

THIMONT JACK Maddy, «Common Agricultural Policy », Institute for Government, 17 mars 2020, https://www.instituteforgovernment.org.uk/explainers/common-agricultural-policy, consulté le 2 septembre 2020.

WILliams Charlotte et MOoNEY Gerry, « Decentring Social Policy? Devolution and the Discipline of Social Policy : A Commentary », Journal of Social Policy, n³7/3, 2008, p. 489-507.

\section{NOTES}

1. ESPING-ANDERSEN Gøsta, The Three Worlds of Welfare Capitalism, Princeton, Princeton University Press, 1990.

2. MARShall Thomas H., Citizenship and Social Class, Cambridge, Cambridge University Press, 1964 (1950).

3. La dévolution d'une partie du pouvoir exécutif et législatif de Londres vers Édimbourg, Cardiff et Belfast s'est faite de manière différenciée selon les territoires. En Écosse, elle a pris la forme d'un parlement directement élu, assorti d'un pouvoir exécutif responsable devant celui-ci (Scotland Act 1998); au pays de Galles, celle d'une assemblée à laquelle a été assorti un gouvernement (Governement of Wales Act 1998, 2006). En Irlande du Nord, la dévolution des pouvoirs (Northern Ireland Act 1998), s'est faite selon les termes des Accords de Belfast de 1998 (Good Friday Agreement): l'assemblée nord-irlandaise, élue directement, doit fonctionner avec un gouvernement où sont représentés à la fois les unionistes et les nationalistes.

4. Greater London Authority Act 1999 et 2007, Local Government Act 2000 et Cities and Local Government Devolution Act 2016.

5. BIRRELL Derek, The Impact of Devolution on Social Policy, Bristol, Policy Press, 2009, p. 1.

6. Charlotte Williams et Gerry Mooney insistent sur la nature relationnelle des politiques publiques britanniques. WILLIAMs Charlotte et MOONEY Gerry, «Decentring Social Policy? Devolution and the Discipline of Social Policy : A Commentary ", Journal of Social Policy, $n^{\circ} 37 / 3$, 2008, p. 489-507.

7. BIRRELL Derek, The Impact of Devolution on Social Policy, op. cit., p. 17.

8. BIRRELL Derek, The Impact of Devolution on Social Policy, op. cit., p. 43.

9. On pense à l'établissement de plafonds nationaux différenciés pour les frais d'inscription à l'université : en Angleterre et au pays de Galles, celui-ci est fixé à $€ 9,250$, contre $£ 4,030$ en Irlande du Nord, et $£ 1,820$ en Écosse.

10. Welfare Reform (Further Provision) (Scotland) Act 2012. 
11. Les modalités de mise en concurrence des composantes du NHS nord-irlandais ont été réformées en 2009, dans le sens d'une réduction du nombre d'acteurs économiques en jeu, d'une concentration des ressources dans les services médicaux plutôt que dans l'administration, et d'une valorisation de la coopération, plutôt que de la compétition.

12. THIMONT JACK Maddy, "Common Agricultural Policy ", Institute for Government, 17 mars 2020, https://www.instituteforgovernment.org.uk/explainers/common-agricultural-policy, consulté le 2 septembre 2020.

13. BRIEN Philip, UK funding from the $E U$, Briefing Paper $n^{\circ} 7847$, House of Commons Library, 28 novembre 2018, p. 15-16.

14. GREER Alan, «Brexit and Devolution », Political Quaterly, n89/1, 2018, p. 134-138.

15. SARGEANT Jess et KLEMPERER, David, "Devolution : common frameworks and Brexit ", Institute for Governement, 16 mars 2020, https://www.instituteforgovernment.org.uk/explainers/ devolution-common-frameworks-brexit, consulté le 13 avril 2020.

16. BRIEN Philip, UK funding from the EU, op. cit., p. 6.

17. COWIE Graaeme, DE MARS, Sylvia, KELLY, Richard et TORRANCE, David, Constitutional implications of the Withdrawal Agreement legislation, Briefing Paper n08805, House of Commons Library, 20 février 2020.

18. THIMONT JACK Maddy, «Withdrawal Agreement Act », Institute for Governement, 4 février 2020, https://www.instituteforgovernment.org.uk/explainers/withdrawal-agreement-act, consulté le 9 avril 2020.

19. Le développement qui suit est largement basé sur le rapport de la Chambre des Communes à ce sujet. CoWIE Graeme, The status of « retained EU law », Briefing Paper n08375, House of Commons Library, 30 juillet 2019.

20. cousson Anne, Droits de l'homme au Royaume-Uni entre 1998 et 2010 : entre politique nationale et droit international, Thèse de doctorat, Université Sorbonne Paris Cité, 2016, p. 14-16.

21. Lock Tobias, "Human Rights Law in the UK after Brexit", Public Law, novembre 2017, p. 117-134.

22. DE MARS Sylvia, MURRAY Colin, O'DONOGHUE Aoife et WARWICK Ben, Continuing EU citizenship "rights, opportunity and benefits " in Northern Ireland after Brexit, Belfast, Northern Ireland Human Rights Commission, mars 2020.

23. Alors même que la sortie de l'UE n'avait pas encore eu lieu et que la Loi sur l'accord de Sortie de 2020 n'avait pas encore été votée, le parlement britannique avait déjà adopté cinq lois, ne concernant pas la législation européenne incorporée, mais anticipant les effets de la sortie de l'Union en matière de relations internationales.

24. THE QUEEN'S SPEECH, London, Prime Minister's Office, 19 décembre 2019.

25. TAYLOR-GOOBY Peter, «Re-doubling the crises of the welfare state: the impact of Brexit on UK welfare politics », Journal of Social Policy, n46/4, 2017, p. 815-835.

26. JEFFREY Charlie, LODGE Guy et SCHMUECKER Katie, "The devolution paradox », in LODGE Guy et SCHMUeCKeR Katie (dir.), Devolution in Practice 2010, London, Institute for Public Policy Research, 2010, p. 9-31, p. 14.

27. FERGUSON Daniel, « Removal of workers' rights in the new EU (Withdrawal Agreement) Bill », House of Commons Library, 20 décembre 2019, https://commonslibrary.parliament.uk/brexit/ legislation/workers-rights-and-the-new-eu-withdrawal-agreement-bill/, consulté le 9 avril 2020. 28. PICKARD Jim et BRUNSDEN Jim, "Fears rise over post-Brexit workers' rights and regulations ", The Financial Times, 25 octobre 2019, https://www.ft.com/content/5eb0944e-f67c-11e9-9ef3eca8fc8f2d65, consulté le 9 avril 2020.

29. JozePA Ilze, fella Stefano, BRowning Steve, SEely Antony, FERGUSON Daniel, SMitg Louise et PRIESTLEY Sara, The UK-EU future relationship negociations: Level playing field, Briefing Paper $n^{\circ} \mathrm{CPB}$ 8852, House of Commons Library, 17 mars 2020. 
30. Voir par exemple les déclarations de Michel Barnier, qui mène les négociations du côté européen, dans cet article du Monde : Ducourtieux Cécile et PetitjeAn Sophie, « Négociations du Brexit : Londres prêt à tout face à l'UE », Le Monde, 26 février 2020, https://www.lemonde.fr/ international/article/2020/02/26/negociations-du-brexit-londres-pret-a-tout-face-a-l-

ue_6030900_3210.html, consulté le 9 avril 2020.

31. FORD Michael, Workers' rights from Europe: the impact of Brexit, Report for the Trade Union Congress, 10 mars 2016, https://www.tuc.org.uk/research-analysis/reports/workers-rightseurope-impact-brexit, consulté le 11 avril 2020.

32. Voir également le rapport suivant pour une vue d'ensemble : STEWART Kitty, COOPER Kerris et SHUTES Isabel, What does Brexit mean for social policy in the UK ?, Social Policies and Distributional outcomes in a Changing Britain, Research Paper n³, février 2019.

\section{RÉSUMÉS}

Cet article examine les conséquences de la sortie du Royaume-Uni de l'Union européenne (UE) sur le régime social britannique, entendu comme l'ensemble complexe formé par les institutions sociales, leurs effets et leurs déterminants. En particulier, le rapatriement des compétences européennes, l'intégration et la réforme de l'acquis communautaire, et la rupture avec les institutions européennes posent des questions de gouvernance et d'équilibres politiques, car ces développements surviennent dans un paysage constitutionnel marqué par l'absence codification et par la dévolution d'une partie du pouvoir exécutif et législatif aux nations. Hors de l'UE, la différenciation des régimes sociaux anglais, écossais, gallois et nord-irlandais est donc vouée à s'accentuer. Hors de l'UE, le Royaume-Uni devra mettre au point de nouveaux mécanismes de lutte contre les inégalités régionales, sous peine de voir celles-ci s'accentuer. Hors de l'UE, le Royaume-Uni renonce également à la protection des droits sociaux par la Cour de justice de l'Union européenne, et ceux-ci relèvent à nouveau de la souveraineté parlementaire. Hors de l'UE, le régime social britannique est donc plus que jamais le produit de rapports de force conjoncturels, marqués notamment par la tentation de la concentration des pouvoirs à Londres, l'héritage conservateur en matière de politiques sociales, et les logiques de différenciation territoriale. Enfin, le processus de sortie de l'UE provoque, en parallèle de cette politisation des questions sociales, un déficit de lisibilité quant à leur définition à moyen et long terme.

This article considers the effects of the United Kingdom's exit from the European Union on the British welfare regime, in other words the country's social institutions, together with their effects and determiners. In particular, the repatriation of European competences, the integration and reform of the acquis communautaire, and the break from European institutions raise questions of governance and political equilibria, since these developments take place against a constitutional backdrop characterized by an absence of codification as well as the devolution of some executive and legislative powers to the British nations. After Brexit, the differentiation between the English, Scottish, Welsh and Northern Irish welfare regimes is therefore bound to increase. The United Kingdom will also have to come up with new mechanisms in order to mitigate regional inequalities, lest these keep growing. Outside of the EU, social rights are no longer protected by the European Court of Justice, and are back within the remit of parliamentary sovereignty. The British welfare regime is thus more than ever subjected to conjunctural balances of power, these being marked, by, among other things, the temptation of 
power concentration in London, the conservative inheritance in terms of social policy, and logics of territorial differentiation. Last but not least, the process of exiting the EU provokes, jointly with its enhanced politicization, a lack of visibility as to the definition of British social policy in the medium and longer term.

INDEX

Keywords : social policy, Brexit, welfare state, European Union, devolution

Mots-clés : politiques sociales, Brexit, welfare state, Union européenne, dévolution

\section{AUTEUR}

\section{CLÉMENCE FOURTON}

Laboratoire CECILLE, Sciences-Po Lille

Clémence Fourton est maîtresse de conférences à Sciences-Po Lille en études anglophones. Sa thèse a porté sur les évolutions du régime social britannique entre 2008 et 2015 . Elle a publié plusieurs articles sur les politiques sociales et les mobilisations collectives outre-Manche, et travaille actuellement à un ouvrage portant sur nos idées reçues au sujet du Royaume-Uni, à paraître aux éditions du Cavalier Bleu. 